Strength training and metabolic conditioning for female youth and adolescent soccer players

\begin{abstract}
Despite the rapid growth in women and girls playing soccer evidence based guidelines to inform conditioning for girls are sparse. The majority of research on youth soccer is conducted in males, which may not be transferable to practice in female teams. This paper reflects on the current evidence base and draws upon the authors' experiences to provide guidelines to improve conditioning programs throughout maturation in girls. The article focuses on the integration of neuromuscular and metabolic conditioning methods to safely improve physical performance and reduce injury risk factors. Guidelines are presented for players pre, circum, and post peak height velocity.
\end{abstract}

\title{
Introduction
}

Women's soccer is growing rapidly in popularity with increasing participation rates worldwide $(10,13)$. There are now opportunities for players to become professional in both the United States of America and Europe. As such there is an increasing demand for strength and conditioning coaches to work with youth players. However, research has often focused upon male soccer players and there is less known about the development of physical qualities in young females throughout maturation. Whilst long-term athlete development models provide guidance for the strength and conditioning coach, these are based more on theoretical concepts rather than empirical evidence (16). Furthermore, rarely in the authors experiences are conditions optimal to allow for the simple application of evidenced based guidelines. In reality time is often limited and participation in supplementary strength and conditioning training can be a new concept to players, parents and coaches. We have found it takes time and education to build a culture of compliance with strength and conditioning training (62). Issues regarding the translation of scientific data to shape and drive practice in soccer have been questioned previously (11). This is evident in the compliance of female soccer players to neuromuscular training programs which can improve physical performance $(15,43,45)$ and reduce injury risk factors $(12,44,50,51,57,59)$ when implemented successfully. Studies, however, often report poor compliance with the training intervention $(1,12,44,59)$ or poor intervention fidelity (29). For example one study demonstrated neuromuscular training improved star excursion balance score yet $31 \%$ of players had to be excluded as they did not comply with the intervention (15). Practitioners working in girls' soccer need to blend evidencedbased practice with practice-based evidence to be successful. This article aims to outline recommendations for practitioners based upon the authors' experience in developing strength and conditioning programs within an English FA girls' center of excellence for players from $9-17$ years of age.

\section{Needs analysis}

A successful match outcome is largely determined by tactical and technical qualities. However, physical attributes such as high-intensity running performance, repeatedsprint ability and speed clearly contribute to elite performance $(17,18,36)$. As a 
general rule more successful teams perform more high intensity running in matches although the nature of repeated high intensity running and sprinting in elite players is highly variable (18). The frequency, duration, intensity, and rest duration of these repeated efforts are unpredictable, as is the nature of neuromuscular load and musculoskeletal strain from changes of direction or explosive jumping movements required within play. Thus, a broad range of physical qualities including neuromuscular (strength, power, balance and proprioception), aerobic, and anaerobic qualities should be developed in female players.

It appears that girls' exhibit a plateau in the development of most physical qualities compared to boys post puberty, with the exception of flexibility $(7,32,56)$. Mujika et al., (37) suggested that female players exhibit lower soccer-specific fitness with a battery of tests including counter movement jump, speed over $15 \mathrm{~m}$, agility, and the Yo-Yo intermittent recovery test. Interestingly, moderate correlations were observed between the counter movement jump and yo-yo test in females, but not in males. It is possible that anaerobic or neuromuscular fitness is of even greater relevance to performance in female players. Moreover, a neuromuscular deficit occurs post puberty that has been associated with increased risk of knee ligament injuries (23), but can be attenuated through appropriate conditioning (50). Injury incidence rates in girls are relatively high in soccer and multifaceted neuromuscular training is essential for female players (5).

Participation in soccer alone is unlikely to guarantee that suitable levels of neuromuscular strength and co-ordination have been developed and may put players at greater risk of injury $(5,14)$. The development of fundamental movements skills, strength, power, speed and agility as early as possible in a players' development may reduce injury and improve athletic performance. Success requires the careful integration of neuromuscular and sport specific fitness training into the players' development. In our experience, periodization of training is ineffective in this environment where we have found higher within player, than between player, variations in weekly training load with a large proportion of training occurring outside of our planned soccer or conditioning sessions (53). Undoubtedly, factors such as transportation, access to facilities and other commitments including schoolwork and examinations can impede on the players' ability to complete planned training and subsequent recovery. Training should be prescribed with an understanding of the athlete's physical capability (fundamental movement skills and soccer specific fitness), biological maturation, and acute readiness to train.

\section{Neuromuscular Training}

Neuromuscular training methods successfully reduce injury in female youth athletes (50), but may also improve soccer specific physical performance. Given poor compliance associated with neuromuscular training programmes for injury reduction it may also be prudent to consider ancillary performance benefits of this training to promote athlete motivation and buy-in (1). Low-level plyometric exercise has been shown to enhance vertical jump height and kicking force in adolescent female players (45) while significant improvements in athletic performance measures have been demonstrated after a 6-week neuromuscular training program (43). A 10-week strength and plyometric programme improved soccer specific endurance and speed beyond traditional aerobic training in high school female soccer players, after 
controlling for marked differences between the groups at baseline (48). Similarly, unpublished data from our practice show longitudinal improvements in speed and acceleration, after controlling for maturation. Furthermore, in a recent case study, the results indicated likely large improvements in repeated sprint performance when neuromuscular training was performed during a return-to-play protocol (60). In Table 1 we provide some guidelines for planning neuromuscular training in girl's soccer teams.

Table 1 here

As aforementioned, it is important to understand both the players' stage of biological maturation and their movement competence prior to designing a training program. Biological age can be calculated simply as a maturity offset (34) and categorized as pre, circum, or post peak height velocity (PHV). Previously, the authors have utilized elements of the Functional Movement Screen $^{\text {TM }}$ (FMS ${ }^{\text {TM}}$ ) and dynamic jump assessments such as Drop Jump (22) and Tuck Jump (39) to assess movement skills and provide a basis for programming. However, a clear maturation effect is present in the FMS ${ }^{\text {TM }}$ (31) and its sensitivity to assess change in youth populations has been questioned (61). Therefore, we propose an alternative approach to assess movement competence through a curriculum (Table 1). This is similar to the model for assessing the progression of athletic movement patterns throughout athletic development proposed by McKeown et al., (33). To reduce the burden of testing on players and the potential for day-to-day variability in execution of an FMS ${ }^{\mathrm{TM}}$ test in youths we propose that exercises incorporated within training are used for testing and monitoring. These should cover the ability to squat, lunge, push, pull, brace, jump and land, and provide relevant information to inform and individualize programs based on what a player can do rather than identify dysfunctions. Figure 1 shows the example for the squat pattern based upon a progression model proposed by Giles (20) (simple to complex, static to dynamic, unloaded to loaded, slow to fast). Table 2 identifies key progressions we have included within our curriculum which provide a framework for integrated neuromuscular training progression. The curriculum incorporates strength, balance and proprioception, proximal control and plyometric exercise, as recommended (50).

Figure 1 here

Table 2 here

\section{Long-term athlete development}

Integrative neuromuscular training is ideally initiated in early adolescents with the most profound effects occurring at younger ages $(41,42)$. Training pre-PHV should aim to improve neuromuscular strength, fundamental movement skills, and speed (30). Exercises that require the body to work through a full range of motion or those incorporating a range of jumping, landing or sprinting tasks in a fun and engaging way are ideal. Animal walks may engage children in these movements and an example may include a "duck walk" (Video 1). This is also an ideal opportunity to introduce a variety of ground-based movements, as shown in Videos 2-4. These can be progressed and incorporated into conditioned games to elicit multi-planar movement and practitioners can manipulate the environment to elicit desired movement outcomes. 
Given appropriate coaching and adequate recovery, advanced training methods such as plyometric training or Olympic-style weight lifting movements are perfectly legitimate training methods pre-PHV but the focus should be on execution of the skill rather than upon the external load lifted. If in doubt, we would advise coaches to undertrain rather than risk over training here. We also suggest that these movements can be incorporated in many different ways and have used "gorilla" and "eagle" positions to elicit triple extension patterns and this concept can be extrapolated to teach full Olympic lifting movements (e.g. figure 2, Video 5). In this example the "gorilla" refers to the starting position of the snatch, and the "eagle", the top of the triple extension. These videos provide examples of ways a strength and conditioning coach can engage children with neuromuscular training, but coaches should be encouraged to use their imagination and work with their players to develop buy-in to the training.

Players circum-PHV may demonstrate reduced co-ordination and joint ranges of motion concurrent with increases in body mass and lever length resulting in greater torque and subsequent injury potential at the knee (23). Thus, in the absence of a neuromuscular spurt seen in males (22), appropriate muscle strengthening is required to improve force absorption and increase joint stability. However, these players need to maintain their mobility and may require simplified exercise prescription. De-loaded (less than body weight) squat variations can decrease the joint loading whilst still providing a training stimulus and may be ideal for a player going through a growth spurt. We have employed the use of functional isometric training (25) to help provide a greater training stimulus safely in this group. Both de-loaded and isometric squat variations have been recommended previously and an additional emphasis on stability and mobility within the regressed movement skill would also be appropriate for this age group (28).

Strength and power development maybe particularly important from an injury prevention perspective in post-pubertal girls (50). We would recommend a progressive neuromuscular and hypertrophy training program which seeks to improve lower limb strength (30) and as such appropriately loaded squat progressions (e.g. over head, front, or single leg squats) at this stage (41). Given technical competency progression to Olympic-style weight lifting may bring about desirable adaptions to ligament thickness and timing of muscle contractions in jumping and landing tasks $(2,21)$. Appropriate hamstring strengthening exercises should also be included as part of a balanced program (40), particularly given the potential relationship between hamstring strength and landing control (58). Movement skill mastery and exercise progressions should be individualized, so the above examples serve as general guidelines only.

Figure 2 here

Figure 3 here

\section{Soccer specific fitness (metabolic-conditioning)}

Metabolic conditioning refers to training targeting the development of both aerobic and anaerobic energy systems and should be specific to the physiological stresses experienced in match play (19). In women's soccer players, match high intensity 
running performance is important as it can differentiate levels of competition (36). A strong relationship exists between match high intensity running and both aerobic capacity, and performance on the yo-yo level 1 recovery test in female players (27). Anaerobic measures such as speed, or counter movement jump height are also important to repeated, short, high intensity activities (56) that typify a soccer match (18). Thus development of both aerobic and anaerobic energy systems appears important in soccer.

The yo-yo test can differentiate between junior (age $17.3 \pm 1.6$ ) and senior female soccer players (37), but the development of soccer specific metabolic conditioning in girls throughout maturation is less understood. Current guidelines (4) suggest children and adolescents are trainable in all fitness components, including aerobic endurance, throughout maturation with limited evidence of a period of enhanced trainability (4). However, given the plateau in endurance performance after the onset of puberty in girls (7) and the increased injury risk associated with both fatigue and changes in body composition (23), metabolic conditioning is particularly relevant for circum- and post-pubertal players.

Debate often surrounds the modality of metabolic conditioning in soccer, in particular between the use of soccer specific small side games or traditional interval training. Data from our practice indicates that players pre-peak height velocity (PHV) do not respond to traditional high intensity or sprint interval training, possibly due to a lower glycolytic capacity to supply ATP during high intensity exercise (6). However, the same protocols provide an appropriate stimulus in those who are circum- or postPHV. In contrast, small sided games alone to do not provide adequate intensity in women soccer players (17). Given the increased neuromuscular load in small-sided games, these should be considered with caution in post-pubescent girls and if they are included to be progressively introduced in-line with the players' technical and physical competence.

Alternatively, fun fast activity blasts (55), that incorporate intense game activities followed by short periods of rest, appear to be best suited to young players (e.g. those pre-PHV). This better resembles how young children actually move and play. Examples could include conditioned games of tag or relay races designed to elicit a high intensity response. Such activities can be progressed and should include variety (38). These training methods have successfully improved markers of health and physical activity and elicited high intensity responses consistently $(54,55)$. The use of small-sided games should provide a further cognitive stimulus and may improve compliance or engagement. This type of training can be incorporated into soccer sessions or provided within an additional strength and conditioning session targeting both metabolic conditioning and neuromuscular training techniques. An example of a 60-minute strength and conditioning session for pre-pubescent girls is presented in Table 1. To maximally utilize the players' ability to learn the session is split into short periods alternating the demands from activities that are cognitively demanding with those that are more physically demanding whilst maintaining the same learning outcome for the session (47).

Table 3 here 
In summary it appears that girls' soccer players require a varied metabolic conditioning stimuli based upon their individual requirements. Coaches working with teams of players across maturation groups may need to be creative in the way they deliver metabolic conditioning to produce positive adaptations throughout. Repeated sprint training methods could provide an adaptable tool for coaches within this age group given the short and sharp nature of the stimulus and potential benefits on neuromuscular and metabolic fitness (52).

\section{Considerations for planning and delivery}

Implementation of training guidelines is unique depending on the environmental, social and psychological nature of the soccer team. This context is critical to the translation of scientific data into applied practice (11). Neuromuscular training maybe ideally suited to dedicated sessions planned within a training week yet practitioners can be limited to a 15-20 minute warm-up period to deliver their intervention. Specific warm-up protocols such as the FIFA11+ or the COREPAC (8) run prior to soccer training could be a time efficient solution but only if barriers to compliance can be overcome $(3,29,44,49,57)$.

Irrespective of the intervention practitioners should ensure the coaching and progression of exercise is sufficient to continue to drive adaptations (44). To promote compliance we recommend a focus upon the education of coaches (24) and players to the potential benefits from both an injury prevention and physical performance enhancement perspective, as the latter may increase player motivation (1).

Periodization of training is difficult given the lack of control over players' additional training and sports demands. Given current evidence on late specialization (35), variety in young players training should be encouraged. Conversely, careful planning of the design of training programs requires greater innovation and monitoring to be incorporated into busy schedules without risking overload. The planning model proposed by Kiely (26) provides a framework for this approach. The use of simple monitoring tools such as RPE and wellness ratings are ideal to use within this model (9) and may provider greater sensitivity to objective measures (46).

\section{Summary and practical applications}

Strength and conditioning coaches working in girls' soccer may need to be adaptable, but neuromuscular training and metabolic conditioning can be implemented to improve physical performance and reduce injury risk factors at all ages. However, the focus and methods used to develop fitness should be specific to a player's maturation and individual needs. Initiating neuromuscular training as early as possible in player's athletic development is recommended and young players will adapt to fun activities targeting specific adaptations. Further prospective research tracking the longitudinal response of girls to structure training interventions throughout maturation would be beneficial.

Take home messages: 
- Integrating neuromuscular and metabolic conditioning has wide spanning benefits for girls' soccer players.

- Movement curriculums provide a framework for individual progressions and regressions of neuromuscular training in a team setting.

- Metabolic and neuromuscular conditioning should be adapted appropriately based upon maturation. 


\section{References:}

1. Alentorn-Geli, E, Myer, GD, Silvers, HJ, Samitier, G, Romero, D, LázaroHaro, $\mathrm{C}$, and Cugat, R. Prevention of non-contact anterior cruciate ligament injuries in soccer players. Part 2: A review of prevention programs aimed to modify risk factors and to reduce injury rates. Knee Surg Sports Traumatol Arthrosc 17: 859-879, 2009.

2. Arabatzi, F and Kellis, E. Olympic weightlifting training causes different knee muscle-coactivation adaptations compared with traditional weight training. J Strength Cond Res 26: 2192-2201, 2012.

3. Attar, Al, WSA, Soomro, N, Pappas, E, Sinclair, PJ, and Sanders, RH. How Effective are F-MARC Injury Prevention Programs for Soccer Players? A Systematic Review and Meta-Analysis. Sports Med 1-13, 2015.

4. Barker, A, Lloyd, RS, Buchheit, M, Williams, C, and Oliver, JL. The BASES expert statement on trainability during childhood and adolescence. The Sport and Exercise Scientist 41: 22-23, 2014.

5. Bergeron, MF, Mountjoy, M, Armstrong, N, Chia, M, Côté, J, Emery, CA, Faigenbaum, A, Hall, G, Kriemier, S, Léglise, M, Malina, RM, Pensgaard, AM, Sanchez, A, Soligard, T, Sundgot-Borgen, J, van Mechelen, W, Weissensteiner, JR, and Engebretsen, L. International Olympic Committee consensus statement on youth athletic development. Br J Sport Med 49: 843-851, 2015.

6. Boisseau, $\mathrm{N}$ and Delamarche, P. Metabolic and hormonal responses to exercise in children and adolescents. Sports Med 30: 405-422, 2000.

7. Catley, MJ and Tomkinson, GR. Normative health-related fitness values for children: analysis of 85347 test results on 9-17-year-old Australians since 1985. Br J Sport Med 47: 98-108, 2013.

8. Celebrini, RG, Eng, JJ, Miller, WC, Ekegren, CL, Johnston, JD, and MacIntyre, DL. The effect of a novel movement strategy in decreasing ACL risk factors in female adolescent soccer players.J Strength Cond Res 26: 3406-3417, 2012.

9. Coutts, AJ. In the age of technology, Occam's razor still applies. IJSPP 9: 741, 2014.

10. Datson, N, Hulton, A, Andersson, H, Lewis, T, Weston, M, Drust, B, et al. Applied physiology of female soccer: an update. Sports Med 44: 1225-1240, 2014.

11. Drust, B and Green, M. Science and football: evaluating the influence of science on performance. J Sports Sci 31: 1377-1382, 2013. 
Mechelen, W. Neuromuscular training injury prevention strategies in youth sport: a systematic review and meta-analysis. Br J Sport Med 49: 865-870, 2015.

13. Fahmy, M. Increase participation and competitions. 5th FIFA women's football symposium FIFA, 2011.

14. Faigenbaum, AD and Myer, GD. Exercise deficit disorder in youth: play now or pay later. Curr Sports Rep 11: 196-200, 2012.

15. Filipa, A, Byrnes, R, Paterno, MV, Myer, GD, and Hewett, TE. Neuromuscular training improves performance on the star excursion balance test in young female athletes. J Orthop Sports Phys Ther 40: 551-558, 2010.

16. Ford, P, De Ste Croix, M, Lloyd, R, Meyers, R, Moosavi, M, Oliver, J, Till, $\mathrm{K}$, and Williams, $\mathrm{C}$. The long-term athlete development model: physiological evidence and application. J Sports Sci 29: 389-402, 2011.

17. Gabbett, TJ and Mulvey, MJ. Time-motion analysis of small-sided training games and competition in elite women soccer players. $J$ Strength Cond Res 22: 543-552, 2008.

18. Gabbett, TJ, Wiig, H, and Spencer, M. Repeated high-intensity running and sprinting in elite women's soccer competition. Int J Sports Phys Ther 8: 130-138, 2013.

19. Gamble, P. Challenges and Game-Related Solutions to Metabolic Conditioning for Team Sports. Strength Cond J, 2007.

20. Giles, K, Penfold, L, and Giorgi, A. A Guide to Developing Physical Qualities in Young Athletes. Movement Dynamics Pty Ltd. Austrailia, 2005.

21. Grzelak, P, Podgorski, M, Stefanczyk, L, Krochmalski, M, and Domzalski, M. Hypertrophied cruciate ligament in high performance weightlifters observed in magnetic resonance imaging. Int Orthop 36: 1715-1719, 2012.

22. Hewett, TE, Myer, GD, Ford, KR, and Slauterbeck, JR. Preparticipation physical examination using a box drop vertical jump test in young athletes: the effects of puberty and sex. Clin J Sport Med 16: 298-304, 2006.

23. Hewett, TE, Myer, GD, Ford, KR, Paterno, MV, and Quatman, CE. The 2012 ABJS Nicolas Andry Award: The sequence of prevention: a systematic approach to prevent anterior cruciate ligament injury. Clin Orthop Relat Res 470: 2930-2940, 2012.

24. Joy, EA, Taylor, JR, Novak, MA, Chen, M, Fink, BP, and Porucznik, CA. 
Factors Influencing the Implementation of Anterior Cruciate Ligament Injury Prevention Strategies by Girls Soccer Coaches. J Strength Cond Res 27: 2263-2269, 2013.

25. Keogh, JWL, Wilson, GJ, and Weatherby, RP. A Cross-Sectional Comparison of Different Resistance Training Techniques in the Bench Press. J Strength Cond Res 13: 247-238, 1999.

26. Kiely, J. Periodization paradigms in the 21st century: evidence-led or tradition-driven? IJSPP 7: 242-250, 2012.

27. Krustrup, P, Mohr, M, Ellingsgaard, H, and Bangsbo, J. Physical Demands during an Elite Female Soccer Game: Importance of Training Status. Med Sci Sports Exerc 37: 1242-1248, 2005.

28. Kushner, AM, Brent, JL, Schoenfeld, BJ, Hugentobler, J, Lloyd, RS, Vermeil, A, et al. The Back Squat: Targeted Training Techniques to Correct Functional Deficits and Technical Factors That Limit Performance. Strength Cond J 37: 13-60, 2015.

29. Lindblom, H, Waldén, M, Carlfjord, S, and Hägglund, M. Implementation of a neuromuscular training programme in female adolescent football: 3-year follow-up study after a randomised controlled trial. Br J Sport Med 48: 1425-1430, 2014.

30. Lloyd, RS and Oliver, JL. The Youth Physical Development Model: A New Approach to Long-Term Athletic Development. Strength Cond J 34: 61-72, 2012.

31. Lloyd, RS, Oliver, JL, Radnor, JM, Rhodes, BC, Faigenbaum, AD, and Myer, GD. Relationships between functional movement screen scores, maturation and physical performance in young soccer players. J Sports Sci 33: 11-19, 2015.

32. Malina, R, Sławinska, T, Ignasiak, Z, and Rożek, K. Sex differences in growth and performance of track and field athletes 11-15 years. $J$ Hum Kinet 24: 79-85, 2010.

33. McKeown, I, Taylor-McKeown, K, Woods, C, and Ball, N. Athletic ability assessment: a movement assessment protocol for athletes. Int J Sports Phys Ther 9: 862-873, 2014.

34. Mirwald, RL, Baxter-Jones, ADG, Bailey, DA, and Beunen, GP. An assessment of maturity from anthropometric measurements. Med Sci Sports Exerc 34: 689-694, 2002.

35. Moesch, K, Elbe, AM, Hauge, MLT, and Wikman, JM. Late specialization: the key to success in centimeters, grams, or seconds (cgs) sports. Scand J Med Sci Sports 21: e282-e290, 2011. 
Match activities of elite women soccer players at different performance levels. J Strength Cond Res 22: 341-349, 2008.

37. Mujika, I, Santisteban, J, Impellizzeri, FM, and Castagna, C. Fitness determinants of success in men"s and women"s football. J Sports Sci 27: 107-114, 2009.

38. Myer, GD, Faigenbaum, AD, Ford, KR, Best, TM, Bergeron, MF, and Hewett, TE. When to initiate integrative neuromuscular training to reduce sports-related injuries and enhance health in youth? Curr Sports Rep 10: 155-166, 2011.

39. Myer, GD, Ford, KR, and Hewett, TE. Tuck Jump Assessment for Reducing Anterior Cruciate Ligament Injury Risk. Athl Ther Today 13: 39-44, 2008.

40. Myer, GD, Ford, KR, Barber Foss, KD, Liu, C, Nick, TG, and Hewett, TE. The relationship of hamstrings and quadriceps strength to anterior cruciate ligament injury in female athletes. Clin J Sport Med 19: 3-8, 2009.

41. Myer, GD, Lloyd, RS, Brent, JL, and Faigenbaum, AD. How Young is "Too Young" to Start Training? ACSMs Health Fit J 17: 14-23, 2013.

42. Myer, GD, Sugimoto, D, Thomas, S, and Hewett, TE. The influence of age on the effectiveness of neuromuscular training to reduce anterior cruciate ligament injury in female athletes: a meta-analysis. $\mathrm{Am} \mathrm{J}$ Sports Med 41: 203-215, 2013.

43. Noyes, FR, Barber-Westin, SD, Tutalo Smith, ST, and Campbell, T. A training program to improve neuromuscular and performance indices in female high school soccer players. J Strength Cond Res 27: 340-351, 2013.

44. Paszkewicz, J, Webb, T, Waters, B, Welch McCarty, C, and Van Lunen, $B$. The effectiveness of injury-prevention programs in reducing the incidence of anterior cruciate ligament sprains in adolescent athletes. J Sport Rehabil 21: 371-377, 2012.

45. Rubley, MD, Haase, AC, Holcomb, WR, Girouard, TJ, and Tandy, RD. The Effect of Plyometric Training on Power and Kicking Distance in Female Adolescent Soccer Players. J Strength Cond Res 25: 129-134, 2011.

46. Saw, AE, Main, LC, and Gastin, PB. Monitoring the athlete training response: subjective self-reported measures trump commonly used objective measures: a systematic review. Br J Sport Med 1-13, 2015.

47. Schmidt, RA. Motor Learning \& Performance. From Principles to Practice. Human Kinetics Books. Champaign, Illinois pp 172$174,1991$. 
48. Siegler, J, Gaskill, S, and Ruby, B. Changes Evaluated in Soccer-Specific Power Endurance Either With or Without a 10-Week, In-Season, Intermittent, High-Intensity Training Protocol. J Strength Cond Res 17: 379-387, 2003.

49. Steffen, K, Myklebust, G, Olsen, OE, Holme, I, and Bahr, R. Preventing injuries in female youth football--a cluster-randomized controlled trial. Scand J Med Sci Sports 18: 605-614, 2008.

50. Sugimoto, D, Myer, GD, Foss, KDB, and Hewett, TE. Specific exercise effects of preventive neuromuscular training intervention on anterior cruciate ligament injury risk reduction in young females: meta-analysis and subgroup analysis. Br J Sport Med 49: 282-289, 2015.

51. Sugimoto, D, Myer, GD, McKeon, JM, and Hewett, TE. Evaluation of the effectiveness of neuromuscular training to reduce anterior cruciate ligament injury in female athletes: a critical review of relative risk reduction and numbers-needed-to-treat analyses. Br J Sport Med 46: 979-988, 2012.

52. Taylor, J, Macpherson, T, Spears, I, and Weston, M. The Effects of Repeated-Sprint Training on Field-Based Fitness Measures: A MetaAnalysis of Controlled and Non-Controlled Trials. Sports Med 1-11, 2015.

53. Taylor, JM, Wright, MD, Hurst, C, and Best, R. Contribution of planned and unplanned training to overall load in elite youth female football players. World Congress of Science in Soccer, Copenhagen, Denmark 23rd-24th May 2015.

54. Taylor, KL, Weston, M, and Batterham, AM. Evaluating intervention fidelity: an example from a high-intensity interval training study. PLoS ONE 10: e0125166, 2015.

55. Taylor, KL. Project FFAB (Fun Fast Activity Blasts): Effect of a novel school-based high-intensity interval training intervention on cardiometabolic risk markers and physical activity levels in adolescents. PhD Thesis, Teesside University, 2014.Available from: http://tees.openrepository.com/tees/handle/10149/337599

56. Vescovi, JD, Rupf, R, Brown, TD, and Marques, MC. Physical performance characteristics of high-level female soccer players 1221 years of age. Scand J Med Sci Sports 21: 670-678, 2010.

57. Waldén, M, Atroshi, I, Magnusson, H, Wagner, $\mathrm{P}$, and Hägglund, M. Prevention of acute knee injuries in adolescent female football players: cluster randomised controlled trial. Br Med J 344: e3042, 2012. 
58. Wild, CY, Steele, JR, and Munro, BJ. Insufficient hamstring strength compromises landing technique in adolescent girls. Med Sci Sports Exerc 45: 497-505, 2013.

59. Wingfield, K. Neuromuscular training to prevent knee injuries in adolescent female soccer players. Clin J Sport Med 23: 407-408, 2013.

60. Wright, MD and Weston, M. Neuromuscular training during returnto-play in an elite junior soccer player: case report. Prof Strength Conditioning 7-12, 2014.

61. Wright, MD, Portas, MD, Evans, VJ, and Weston, M. The effectiveness of 4 weeks of fundamental movement training on functional movement screen and physiological performance in physically active children. J Strength Cond Res 29: 254-261, 2015.

62. Wright, MD. Neuromuscular training in girl's football - the challenge of applying evidence based practice in an FA center of excellence. $J$ Sports Sci 32: s93-s100, 2014.

\section{Figures}

Figure 1: Squat continuum to assist progression and monitoring of training across young players. This provides a continual assessment tool that can be used throughout training where level one is equivalent to 30 points on the scale and level six 180 points.

Figure 2: Coaching of Olympic lifting derivatives through animal shapes a "gorilla" $b$ "stork" c "eagle" d "gorilla roar". Video 5 demonstrates these combined to develop a snatch pattern. 